DOI: https://doi.org/10.46296/yc.v5i9edespsoct.0129

\title{
ANÁLISIS DE LA ECONOMÍA COLABORATIVA PARA LAS PYMES RURALES DE MANABÍ 2021-2022
}

\section{ANALYSIS OF THE COLLABORATIVE ECONOMY FOR RURAL SMES IN MANABÍ 2021-2022}

\author{
Zambrano-Roldán Karen Estefanía ${ }^{1}$ \\ ${ }^{1}$ Profesora Titular de la Universidad Laica Eloy Alfaro de Manabí. Manta, Ecuador. \\ Correo: karen.zambrano@uleam.edu.ec. ORCID ID: https://orcid.org/0000-0001-9693- \\ 6679
}

\section{Resumen}

En las últimas décadas, ha sido innegable el incremento de la migración rural hacia las zonas urbanas, especialmente en países de América Latina. Esto se debe a la falta de oportunidades laborales, a la ineficacia en la comercialización y la ineficiente gestión gubernamental, generando situaciones críticas desde la perspectiva socioeconómica, cultural y ambiental. En este contexto, los factores empresariales que inciden en el desenvolvimiento de los emprendimientos rurales en el entorno comercial requieren la aplicación de metodologías innovadoras, tecnológicas y tecnificadas que aseguren la calidad en la gestión administrativa. El emprendimiento rural llevado a cabo de forma ineficiente y abrupta solo se convierte en una idea intangible y fugaz que, en su momento, pudo estar motivada por la carencia de factores como un empleo óptimo y bien remunerado, o en esencia, el desempleo, que es el desenlace de la crítica situación que viven los países a nivel global, y en efecto, a nivel nacional el panorama del éxito empresarial resulta mínimamente alentador ya que los emprendimientos e iniciativas emprendedoras se encuentran en pleno crecimiento y continúan en constante ascenso.

Palabras claves: economía, PYMES, gestión administrativa, emprendimiento rural.

\begin{abstract}
In recent years, the increase in rural migration to urban areas, especially in Latin American countries, is undeniable. This is due to the lack of job opportunities, ineffective marketing and inefficient government management, generating critical situations from a socio-economic, cultural and environmental perspective. In this context, the business factors that affect the development of rural enterprises in the commercial environment require the application of innovative, technological and technological methodologies that ensure quality in administrative management. Rural entrepreneurship carried out inefficiently and abruptly only becomes an intangible and fleeting idea that, at the time, could be motivated by the lack of factors such as an optimal and well-paid job, or in essence, unemployment, which It is the outcome of the critical situation that countries are experiencing at the global level, and indeed, at the national level, the panorama of business success is minimally encouraging, since entrepreneurships and entrepreneurial initiatives are in full growth and continue to rise constantly.
\end{abstract}

Keywords: economy, SMEs, administrative management, rural entrepreneurship.

Información del manuscrito:

Fecha de recepción: 02 de julio de 2021.

Fecha de aceptación: 10 de octubre de 2021.

Fecha de publicación: 25 de octubre de 2021. 


\section{Introducción}

Los emprendimientos e iniciativas emprendedoras tienen dos razones esenciales para ser puestos en marcha; en primer lugar, aquellos que son propuestos a partir de las oportunidades que brindan los mercados, o en el mismo caso, por la iniciativa innovadora en base al desarrollo de un efecto diferenciador en la sociedad. Por otro lado, la segunda razón radica en que el emprendimiento se origina por necesidad, ya sea para obtener beneficios económicos, o para solventar las necesidades previstas en una determinada locación (EI Shoubaki et al., 2018; Klepitskij, 2020; Machado et al., 2016). Estas resultan en la motivación más básica de los emprendedores, la misma que en tiempos más remotos, se relacionaba estrictamente con el instinto de supervivencia frente a las amenazas y las dificultades.

La innovación y el emprendimiento se encuentran directamente vinculados al liderazgo y a factores externos, como el caso de las políticas públicas, los proyectos, el factor de inversión, los desafíos, las oportunidades y otros factores que incentivan a concretar la idea de emprender e innovar. Esta temática se origina por la necesidad de motivación de muchas personas con respecto a encontrar una oportunidad que aprovechar, y de este modo, contribuir al desarrollo personal, resultando en una temática de estudio cada vez más compleja y necesaria (Romero \& Restrepo, 2016). Partiendo de ello, Mccaffrey (2017) reconoce que los fundamentos del emprendimiento e innovación son constantes determinadas por los ámbitos de acción y diversos avances del emprendimiento durante los últimos años; también da cuenta del nexo que existe entre el emprendimiento y el factor académico. Como resulta evidente, en el caso de algunos países desarrollados y en América Latina, los emprendedores se encuentran viviendo momentos determinantes con respecto al emprendimiento y la constante de la innovación, surgiendo de este modo, las necesidades de cambios en la sociedad y su entorno.

Para identificar el emprendimiento en el Ecuador, es indispensable analizar al mismo desde su propia historia como conocimiento 
ancestral y tradicional, que en el contexto actual se encuentra en ascenso, entre otras cosas, por los intentos de prevalecer ante los determinismos imperantes que se encontraban presentes hasta la década de 1970 (Samaniego-Erazo, 2014), que limitaba el rol de los hombres y mujeres como agentes de cambio marcados por el sexismo, más aún cuando una parte significativa de la población debía responder a los diferentes cambios de los sistemas, modelos económicos y políticos instaurados en los diferentes gobiernos por los cuales ha pasado el país; así como cuando se reconocen disposiciones concebidas desde los países desarrollados.

\section{La economía colaborativa y las pequeñas y medianas empresas}

El emprendimiento se identifica con el hombre racional por excelencia, del mismo modo con aquellos individuos que con sus actividades desencadenan en estabilidad en los mercados, como agentes dinámicos e innovadores en el desarrollo sustentable. Sin embargo, en la última década, al emprendimiento se le han anexado nuevas categorías, como el caso de la categoría social.
El

reconocimiento del emprendimiento en Ecuador se origina desde el 2004, y más específicamente, desde las aulas universitarias del país, acompañadas de organismos ajenos a la realidad ecuatoriana, como el Global Entrepreneurship Monitor (GEM) y también la Confederación de Empresarios de Madrid (CEIM). Esto lo corrobora Zamora-Boza (2017), quien establece que, en los últimos años, la promoción del emprendimiento ha sido una temática común a nivel mundial, por sus implicaciones en el incremento del índice del empleo, en el crecimiento económico y en la mejora de la calidad de vida. Bajo este enfoque, Ecuador es considerado el segundo país con el mayor número de emprendimientos creados en Latinoamérica, puesto que su tasa de actividad emprendedora temprana (TEA) es del $36,2 \%$; tan solo superado por Chile (36,7\%). Sin embargo, y a pesar de que se han desarrollado diversas medidas y políticas públicas destinadas a explotar la funcionalidad y rentabilidad de estos emprendimientos, el país no cumple con todos los requisitos para generar un ambiente completamente 
dinámico y sostenible de emprendimientos, de manera que la tasa de cierre de negocios alcanza el 9,3\%; esto de acuerdo con el Global Entrepreneurship Monitor (GEM, 2019).

La construcción de emprendimientos y empresas resulta una prioridad cuando se trata de promover el desarrollo socioeconómico de un país; por ello, es imperioso determinar cuál es la contribución de estas organizaciones a los objetivos comunes de la sociedad. El emprendimiento genera un impacto positivo en la construcción de una sociedad sustentable en base a la creación de plazas de trabajo y fuentes de empleo, lo que desencadena en el crecimiento económico de una región o país (lyoha \& Oviasuyi, 2015). El emprendimiento en una característica estructural y primordial de las economías latinoamericanas, en parte, como respuesta a los altos niveles de desempleo que predominan en la región, que a través de iniciativas emprendedoras se convierte en una alternativa eficaz para la producción de ingresos.

Esto lo corroboran autores como Valle \& Fuentes (2016), quienes alegan que una de las principales problemáticas de la economía popular y solidaria la constituyen los débiles procesos de innovación que se presentan en el contexto en el que se desarrollan. Por ello consideran que, en esta línea se deben fomentar y desarrollar las capacidades de los emprendedores, lo cual supone la instrucción de capacidades interpretativas, reflexivas, cognitivas, y comunicacionales que resultan básicas para llevar a cabo este fin. Para solventar esto, los autores proponen diseñar programas y acciones necesarias en su estructura organizacional, lo que permitirá desplegar las potencialidades de desarrollo interno identificadas, así como su integración en los programas regionales, recalcando la importancia de los proyectos e iniciativas emprendedoras locales. Se exponen los pilares fundamentales de la economía popular y solidaria, particularizando a nivel regional el caso de Ecuador, en donde resulta indispensable, en primera instancia, realizar un análisis histórico y comparativo entre el país y el contexto internacional, respecto al origen de empresas e iniciativas emprendedoras. 
Se destaca la importancia de conocer la situación a la que se enfrentan muchos individuos, que al incurrir en la tentativa de tomar las riendas de una aventura empresarial para mejorar sus condiciones de vida, no logran obtener el rendimiento esperado, llegando en muchas ocasiones al fracaso; pese a ello, existe la posibilidad de reconocer oportunidades que ofrece el mercado (Pico- Versoza, 2017), que al ser identificadas oportunamente solidificarán una alternativa que apunte hacia la planificación estratégica y que a la vez pueda posicionar al emprendedor hacia un óptimo desarrollo y éxito empresarial.

En Ecuador, el panorama del éxito empresarial resulta mínimamente alentador ya que los emprendimientos e iniciativas emprendedoras se encuentran en pleno crecimiento y continúan en constante ascenso.

emprendimiento rural llevado a cabo de forma ineficiente y abrupta solo se convierte en una idea intangible y fugaz que, en su momento, pudo estar motivada por la carencia de factores como un empleo óptimo y bien remunerado, o en esencia, el desempleo, que es el desenlace de la crítica situación que viven los países a nivel global, y en efecto, Ecuador no es la excepción. No obstante, estos factores han logrado que los individuos construyan ideas y generen herramientas que permitan viabilizar de forma eficiente la tentativa de empezar un negocio en base a las necesidades del mercado, lo que específicamente en Ecuador, resulta acertado, ya que desencadena en un alto índice de emprendimiento (Prada, 2020). Lo negativo es que la mayoría de los emprendimientos, al ser generados de forma rápida no conllevan una planificación y asesoría que garantice su supervivencia en el mercado a largo plazo. A pesar de no encontrarse suficientemente clara, la temática del emprendimiento de carácter social cuenta con varios aportes teóricos y empíricos que han contribuido a generar una discusión relativamente importante en esta área. La revisión de diversos conceptos ha permitido puntualizar aspectos importantes en la definición del término emprendimiento, así como la diferenciación entre el emprendimiento empresarial y el emprendimiento social (PérezBriceño et al., 2017). Los autores 
coinciden en que el emprendimiento de carácter social está caracterizado principalmente por su enfoque a la resolución y solvatación de problemáticas sociales sin ánimo de lucro, con un fin enteramente solidario.

Particularmente, en este contexto los emprendedores de bases sociales cuentan con las mismas características de liderazgo y acción que un emprendedor ordinario, señalando la peculiaridad de una necesidad intrínseca por aportar en aquellas causas y problemáticas sociales, convirtiéndose de esta manera en un medio oportuno de cambio social. Por otro lado, y con respecto a los emprendimientos sociales, se recalcan las características tanto de organizaciones que sustentan este tipo de emprendimiento como de empresas pioneras en el aprovechamiento de cuestiones sociales, que en su mayoría se desarrollan en entornos rurales como parte de iniciativas de inclusión.

\section{Métodos}

La investigación tuvo un enfoque mixto, dado que se utilizó la recolección de datos para probar hipótesis, con base en la medición numérica y el análisis estadístico, para establecer y probar teorías- en síntesis, se abordó el tema de estudio desde los aspectos cuantitativos y medibles; a fin de identificar hallazgos de importancia que sirvan de base a la investigación. Del mismo modo, se aplicó la investigación teórica bibliográfica, mediante el análisis de textos especializados, respecto al objeto en estudio considerando aquellos producidos durante la última década para tener la información más actualizada. Se implementó una investigación analítica y deductiva que nos ayudó a cómo entender y así poder observar las causas, la naturaleza y los efectos que puede tener esta investigación, estos métodos nos permitieron conocer más el objetivo de estudio que se pueda presentar para así explicar y comprender cada uno de los comportamientos y establecer nuevas teorías. Las técnicas utilizadas en esta investigación consistieron en la 
recolección de datos estadísticos de investigaciones previas para evidenciar los resultados de la investigación.

\section{Resultados}

\section{La economía colaborativa y las PYMES rurales}

A nivel local, Acosta (2016) considera indispensable establecer la situación actual de la actividad comercial y emprendedora, tomando un enfoque específico en el ámbito del sector turístico.

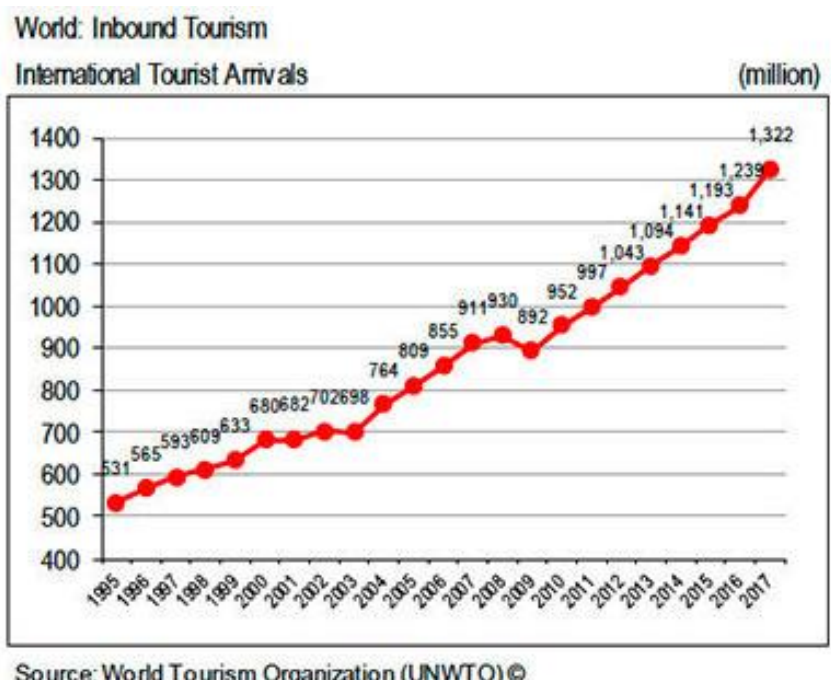

Ilustración 1. Fuente: http://somosmanabi.com/index.php/nuestro-blog/item/manabi-turismogran-oportunidad

En ese sentido, se plantea el objetivo primordial de desarrollar y potencializar el turismo comunitario en la zona rural y, que el mismo sea una tentativa de desarrollo para las localidades aledañas; se ha optado por la oportuna implementación del Plan de Desarrollo Nacional del período 2017, el que establece entre sus objetivos fundamentales: mejorar la calidad de vida de los ciudadanos, lo que según Gavrilović \& Maksimović (2018), es justamente lo que se pretende alcanzar con el minucioso estudio de la actividad turística comunitaria en una determinada localidad rural. Se resalta la importancia de identificar los recursos y atractivos que poseen estas localidades, que, en conjunto con un trato eco sustentable, podrían 
convertirse de forma óptima en potenciales atractivos turísticos para el sector en mención, con réplicas semejantes tanto a nivel cantonal, provincial y nacional. Sin embargo, para lograr este fin resulta completamente necesaria la implementación de directrices que evidencien la viabilidad de potenciar estos lugares como atractivos turísticos, para que de esta manera, las autoridades estatales en conjunto con la comunidad puedan ejercer de manera eficiente la denominada actividad turística comunitaria, partiendo desde tentativas ecológicas sostenibles y con un mínimo impacto en el medio ambiente, para que de esta manera los beneficios económicos sean redistribuidos de manera equitativa y en beneficio de los miembros activos de la localidad, lo que a su vez, facilitará la optimización del ámbito socioeconómico de la provincia de Manabí, desencadenando en un resultado positivo, puesto que resulta evidente el interés de los habitantes de la localidad por edificarse como los actores principales en el desarrollo del turismo eco-comunitarios.

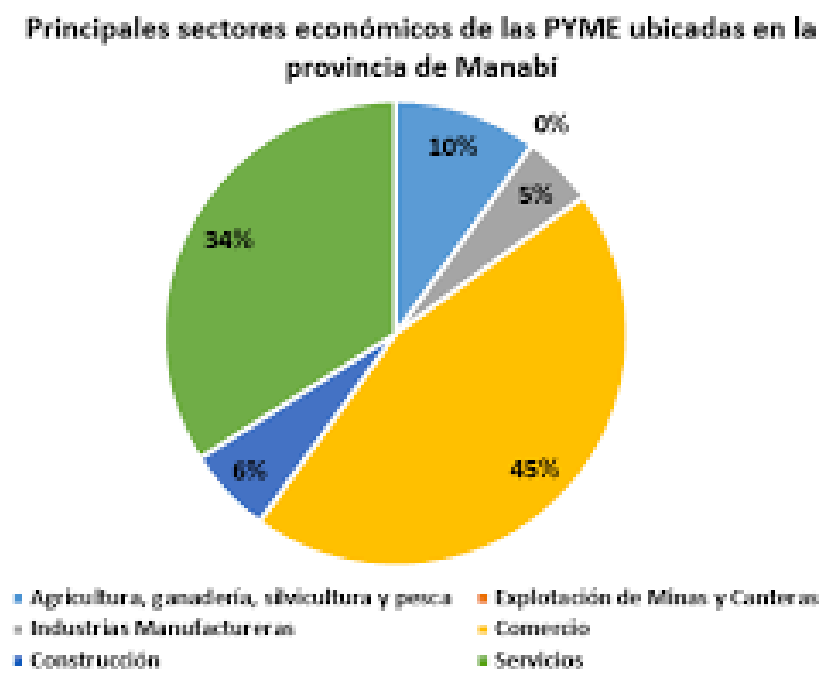

Ilustración 2. Fuente: Alvarado Cedeño, (2019). Modelo de desarrollo utilizando un sistema de información gerencial en la competitividad del sector comercial en la ciudad de Portoviejo.

Universidad Tecnológica Empresarial de Guayaquil.

Los factores económicos también generan un impacto altamente influyente en la sustentabilidad del emprendimiento, puesto que otra falencia del emprendimiento rural local es la gestión administrativa en base a conocimientos empíricos, que, en algunos casos, pueden 
alejarse de los procesos estratégicos

que exige el desarrollo empresarial.

En ese sentido, Prado \& Mishell (2018) analizan el impacto socioeconómico de factores asociados a las microfinanzas en un determinado sector, como auge comercial de la ciudad de Manta.
Dado esto, afirman que, en primer lugar, resulta completamente indispensable entender que el concepto de microfinanzas ha sido una variable que ha ido evolucionando a través del tiempo, a la par el dinamismo con el que han evolucionado las microempresas.

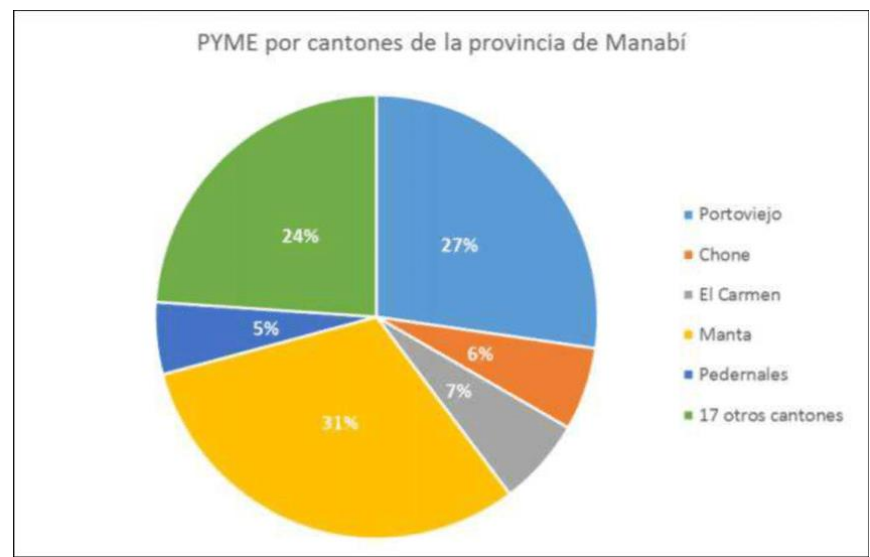

Ilustración 3. Fuente: Palma Pérez, Xiomara. (2018). El marketing sensorial y su influencia en el comportamiento de compra de los consumidores de la ciudad de Manta.

Tal como evidencian los datos obtenidos por Palma (2018), el 31\% de las pequeñas y grandes empresas se localizan en gran medida en la ciudad de Manta. El segundo lugar con el mayor porcentaje de PYMES es la ciudad de Portoviejo con el $27 \%$. El tercer lugar con más incidencia de PYMES lo conforman 17 cantones rurales, que en conjunto tienen en $24 \%$ de las PYMES.

\section{Discusión}

Análisis de la Economía colaborativa para las PYMES rurales de Manabí

El impacto de los programas relacionados con la gestión del talento humano en asociaciones radicadas en el emprendimiento rural a nivel provincial de Manabí, Ecuador ha sido abordado por Villavicencio et al. (2020). Estas cooperativas y asociaciones de investigación se encuentran enfocadas principalmente en 
mantener la formación permanente de su talento humano y potenciales colaboradores, lo que les permite asegurar la consolidación de una iniciativa emprendedora o su idea de negocio y promoviendo de esta forma la posibilidad de consolidarse como asesoría permanente del talento humano. Indiscutiblemente, esta temática se encuentra directamente relacionada con un aspecto vital para la sostenibilidad a nivel territorial, fundamentada en dos constantes íntimamente relacionadas, como son los atractivos turísticos y la posibilidad del desarrollo económico (LoorChavez et al., 2020). Por ello, es fundamental aplicar procedimientos para la recopilación y posterior análisis de la información existente con respecto a los activos más representativos de determinada localidad, como en el caso específico de los atractivos naturales, patrimoniales, culturales, recreativos, que evidentemente se configuran como los principales atractivos locales para los consumidores internos y foráneos, constituyéndose en el soporte económico para iniciativas emprendedoras que dependen en su totalidad de la actividad turística, particularmente en la zona rural; entre las que destacan restaurantes, centros de diversión, hoteles, bares, pequeños comercios, artesanos, comunidad, entre otros. Esto lo corroboran Mosquera-López \& Veintimilla-Palacios (2015), quienes concluyen que la creación de una propuesta de desarrollo turístico para los sectores rurales de la ciudad de Manta, como en el caso de la Parroquia Santa Marianita, tendría un impacto directamente positivo con respecto al desarrollo turístico a nivel de la provincia de Manabí, así como a nivel nacional, además de convertirlo en un popular destino turístico caracterizado por ser un atractivo de sol y playa.

Las estrategias de emprendimiento puestas en marcha por migrantes que regresaron al Ecuador tras haberse radicado durante un largo período de tiempo en países de primera línea con economías desarrolladas como España, tienen afinidad con las decisiones políticas de los países que permiten el regreso de sus migrantes (VegaSolís, 2016). Se detalla la manera en que la política se ha extendido en un contexto de fluctuaciones y conflictos puntuados por la reciente crisis 
económica en España, así como por las reformas políticas, de carácter económico y social a nivel de Ecuador. A partir por ello, cabe resaltar la importancia de discutir desde una perspectiva de género y generación, el factor emprendimiento con respecto a estrategias adoptadas por los ciudadanos retornados, que no ven en los pequeños negocios, microempresas y pequeñas empresas un factor determinante para retornar a su país de origen.

Para emprender un negocio y llevar las riendas de una iniciativa emprendedora, el comportamiento sigue un patrón y una tendencia, que se valora y articula con otros factores de la vida a nivel individual y familiar, entre los que destacan factores afectivos, como la organización social del cuidado, o por otro lado la formación de hijos e hijas, y finalmente otros proyectos de carácter laboral. Dicha articulación debe ser analizada desde diversas perspectivas, que contemplen las dinámicas y tendencias de clase, edad y género en los procesos migratorios, así como el modo en que se despliegan dependiendo de la composición del núcleo familiar.
El factor de la comunicación se presenta como una estrategia generadora de confianza en los emprendimientos, principalmente a razón de la capacidad de influencia que poseen los individuos, así también, la capacidad de generar y mantener la confianza, cuando existen incongruencias entre lo que se dice y hace, o cuando los mensajes se perciben como promesas inalcanzables o con tintes manipuladores (Suarez \& Maldonado, 2018). En este contexto, los emprendimientos son organizaciones con capacidades de adaptación inmediata, para responder a las necesidades cambiantes de los diversos entornos, de modo que es indispensable analizar el factor de la comunicación como estrategia generadora de confianza en los emprendimientos, micro, pequeñas y medianas empresas del sector rural. En esta misma línea, Villacres-Borja (2017) destacan la manera en la que la gestión del emprendimiento influye favorablemente en la competitividad de las micro, pequeñas y medianas empresas a nivel del Ecuador. La gestión eficiente de las Mipymes en relación con la planificación estratégica, la capacitación del 
personal y el conocimiento del mercado influye considerablemente en el factor de la competitividad, además de la supervivencia de dichos emprendimientos en un mercado globalizado y cada vez más competitivo.

\section{Conclusiones}

En las últimas décadas, es innegable el incremento de la migración rural hacia las zonas urbanas, especialmente en países de América Latina. Esto se debe a la falta de oportunidades laborales, a la ineficacia en la comercialización y la ineficiente gestión gubernamental, generando situaciones críticas desde la perspectiva socioeconómica, cultural y ambiental. En este contexto, Los factores empresariales que inciden en el desenvolvimiento de los emprendimientos rurales en el entorno comercial requieren la aplicación de metodologías innovadoras, tecnológicas y tecnificadas que aseguren la calidad en la gestión administrativa. Camino \& Aguilar (2017) exponen que las variables empresariales que se impulsan a raíz de iniciativas de emprendimiento, la incorporación de estrategias innovadoras en su metodología de venta y la observación del impacto de determinadas políticas socioeconómicas benefician directamente a la posición de las empresas en el mercado, destacándose que la determinación de un panorama a través del riguroso análisis de los medios empresariales refiere los pilares fundamentales para la consolidación de las actividades previstas y proyectos empresariales emergentes, así como negocios en surgimiento $y$ principalmente aportes esenciales para garantizar el desarrollo de las pequeñas y medianas empresas.

En concordancia con lo manifestado previamente, es necesario evaluar las experiencias de los emprendedores, en aras de atender sus necesidades y elaborar propuestas que las resuelvan. Barragán \& Ayaviri (2017) destacan la importancia de analizar empíricamente la relación existente entre innovación, emprendimiento y desarrollo local sostenible, para lo cual ejemplifican el desarrollo evidenciado a partir de la implementación de estrategias de emprendimiento de áreas rurales. A 
nivel de la provincia de Manabí, tal como evidencian los datos obtenidos por Palma (2018), el $31 \%$ de las pequeñas y grandes empresas se localizan en gran medida en la ciudad de Manta. El segundo lugar con el mayor porcentaje de PYMES es la ciudad de Portoviejo con el 27\%. El tercer lugar con más incidencia de PYMES lo conforman 17 cantones En este caso de estudio afirman que el emprendimiento contribuye de manera significativa al desarrollo sostenible local en conjunto con tentativas de innovación; de igual forman destacan que la organización comunitaria, la economía solidaria, el liderazgo y la cultura emprendedora son factores clave para el éxito de los emprendimientos en este sector del austro ecuatoriano. Se reporta que el emprendimiento ha contribuido al desarrollo local, generando resultados significativamente beneficiosos para las áreas rurales, tales como la generación de fuentes de empleo que permiten mejorar el nivel de vida de la población basados en principios de equidad, participación en la economía colaborativa y la economía solidaria.

\section{Bibliografía}

Acosta, P. I. (2016). Turismo comunitario: análisis del desarrollo turístico comunitario en la parroquia rural San Lorenzo, cantón Manta, Ecuador. Magazine de las Ciencias: Revista de Investigación e Innovación, 1(1), 19-32.

Barragán, M. C., \& Ayaviri, V. D. (2017). Innovación y Emprendimiento, y su relación con el Desarrollo Local del Pueblo de Salinas de Guaranda, Provincia Bolívar, Ecuador. Información tecnológica, 28(6), 71-80.

Camino, R. C., \& Aguilar, A. E. (2017). Emprendimiento e innovación en Ecuador, análisis de ecosistemas empresariales para la consolidación de pequeñas y medianas empresas. INNOVA Research Journal, 2(9.1), 73-87.

El Shoubaki, A., Laguir, I., \& den Besten, M. (2019). Human capital and SME growth: The mediating role of reasons to start a business. Small Business Economics, 54, 1107-1121. https://doi.org/10.1007/s1118 7-018-0129-y

Gavrilović, Z., \& Maksimović, M. (2018). Green innovations in the tourism sector. Strategic Management, 23(1), 36-42. 
https://doi.org/10.5937/StraM an1801036G.

https://doi.org/10.1016/j.techfore.20 19.119791

lyoha, F., \& Oviasuyi, P. (2015). Entrepreneurship and development. Vol. 1. pp. 0931.

Klepitskij, I. (2020). Starting a Business: Risk of Penalties. Law. Journal of the Higher School of Economics. 130161.

https://doi.org/10.17323/2072 $-8166.2020 .2 .130 .161$.

Loor-Chavez, T. D., VillamarinVillota, W. H., SabandoMendoza, E. R., ArteagaFlores, R. K., Cruz-Mera, R. H., \& Arias-Arias, A. E. (2020). Los atractivos turísticos de Manta y su incidencia en el desarrollo económico de las parroquias urbanas y rurales. Revista Científica FIPCAEC (Fomento de la investigación y publicación en Ciencias Administrativas, Económicas y Contables). Polo de Capacitación, Investigación y Publicación (POCAIP), 5(18), 41-77.

https://doi.org/10.23857/fipca ec.v5i18.200

Machado, H., Gazola, S., Fabrício, J. \& Añez, M. (2016). Women Entrepreneurs: Reasons And Difficulties For Starting In Business. Ram. Revista de Administração Mackenzie, 17,
$15-$ 38. https://doi.org/10.1590/167869712016/administracao.v17 n3p15-38.

Mccaffrey, M. (2017). Extending the Economic Foundations of Entrepreneurship Research: The Economic Foundations of Entrepreneurship. European Management Review. https://doi.org/10.1111/emre. 12158.

Mosquera-López, J. P., \& VeintimillaPalacios, K. V. (2015). Propuesta de desarrollo turístico de la parroquia rural Santa Marianita del cantón Manta, provincia de Manabí.

Pérez-Briceño, J. C., JiménezPereira, S. E., \& GómezCabrera, O. A. (2017). Emprendimiento social: una aproximación teóricapráctica. Dominio de las Ciencias, 3(2), 3-18. https://doi.org/10.23857/pocai $\mathrm{p}$

Pico-Versoza, L. M. (2017). El emprendimiento por necesidad, una ventana hacia el desarrollo de oportunidades de negocios.

Prada, R. (2020). Entrepreneurial attitude and business success. 18.

Prado, C., \& Mishell, H. (2018). Proyecto de Investigación para Determinar el Impacto Socio- Económico de las 
Microfinanzas" Credife" en los emprendimientos de la parroquia Manta en el período 2014-2016

(Doctoral dissertation).

Romero, X. A. V., \& Restrepo, S. O. (2016). Emprendimiento e innovación:

Una aproximación teórica. Dominio de las Ciencias, 2(4), 346-369.

Samaniego, M. P. (2017, June). Emprendimiento $y$ responsabilidad social: Análisis estadístico de la participación de la mujer en Ecuador. In Conference Proceedings (Vol. 1, No. 1).

Samaniego-Erazo, F. (2014). Análisis y perspectiva del emprendimiento empresarial ecuatoriano en el contexto de la política económica del buen vivir. Observatorio de la Economía Latinoamericana, 201, 1-5.

Suarez, L., \& Maldonado, J. (2018). La comunicación como estrategia generadora de confianza en los emprendimientos. INNOVA Research Journal, 3(1), 95107.

Valle, A., \& Tobar, G. (2017). El marketing relacional y la rentabilidad en los servicios prestados por tres concesionarios automotrices en la provincia de Tungurahua del Ecuador. Revista
UNIANDES Episteme, 4(2), 135-149.

Valle, J. A. B., \& Fuentes, N. M. (2016). Gestión de emprendimiento de Economía Popular y Solidaria para potenciar un desarrollo comunitario local. Avances, 18(1), 66-76.

Vega-Solís, C. (2016). El retorno más allá del mito del emprendedor: estrategias económicas, familiares y afectivas de mujeres $y$ hombres a su regreso a Ecuador desde España. Papers: Revista de sociología, 101(4), 0415-449.

Villacres-Borja, R. M. (2017). Gestión del emprendimiento y su impacto en la competitividad de las Mipymes manufactureras de la provincia de Bolívar en el Ecuador.

Villavicencio, D. V. C., Quiroz, C. A. M., Garcia, M. I. G., \& Rosado, V. F. M. (2020). Gestión del talento humano en cooperativas de emprendimiento rural. CIENCIAMATRIA, 6(10), 585597.

Zamora-Boza, C. S. (2017). La importancia del emprendimiento en la economía: el caso de Ecuador. Revista Espacios Económicos, 39(8), 6-7. 